\title{
Alterations of anti-inflammatory lipids in plasma from women with chronic widespread pain - a case control study
}

\author{
Niclas Stensson*, Bijar Ghafouri, Björn Gerdle and Nazdar Ghafouri
}

\begin{abstract}
Background: Chronic widespread pain conditions (CWP) such as the pain associated with fibromyalgia syndrome (FMS) are significant health problems with unclear aetiology. Although CWP and FMS can alter both central and peripheral pain mechanisms, there are no validated markers for such alterations. Pro- and anti-inflammatory components of the immune system such as cytokines and endogenous lipid mediators could serve as systemic markers of alterations in chronic pain. Lipid mediators associated with anti-inflammatory qualities - e.g., oleoylethanolamide (OEA), palmitoylethanolamide (PEA), and stearoylethanolamide (SEA) - belong to $\mathrm{N}$-acylethanolamines (NAEs). Previous studies have concluded that these lipid mediators may modulate pain and inflammation via the activation of peroxisome proliferator activating receptors (PPARs) and the activation of PPARs may regulate gene transcriptional factors that control the expression of distinct cytokines.

Methods: This study investigates NAEs and cytokines in 17 women with CWP and 21 healthy controls. Plasma levels of the anti-inflammatory lipids OEA, PEA, and SEA, the pro-inflammatory cytokines TNF- $\alpha, I L-1 \beta$, IL-6, and $\mathrm{IL}-8$, and the anti-inflammatory cytokine IL-10 were investigated. T-test of independent samples was used for group comparisons. Bivariate correlation analyses, and multivariate regression analysis were performed between lipids, cytokines, and pain intensity of the participants.
\end{abstract}

Results: Significantly higher levels of OEA and PEA in plasma were found in CWP. No alterations in the levels of cytokines existed and no correlations between levels of lipids and cytokines were found.

Conclusions: We conclude that altered levels of OEA and PEA might indicate the presence of systemic inflammation in CWP. In addition, we believe our findings contribute to the understanding of the biochemical mechanisms involved in chronic musculoskeletal pain.

Keywords: Chronic widespread pain, Inflammation, $\mathrm{N}$-acylethanolamines, Palmitoylethanolamide, Oleoylethanolamide, Cytokines

\section{Background}

Chronic widespread pain (CWP), which is often associated with fibromyalgia syndrome (FMS), is a significant health problem. The prevalence of CWP is approximately $10 \%$ $[1,2]$, and the prevalence of FMS is between 2 and $4 \%$ $[3,4]$. The cause of CWP is multifactorial, but the underlying biochemical-neurobiological mechanisms are still unknown. However, both central and peripheral pain

\footnotetext{
* Correspondence: niclas.stensson@liu.se

Pain and Rehabilitation Centre, Department of Medical and Health Sciences, Linköping University, Linköping, Sweden
}

mechanisms influence the clinical presentation of CWP/ FMS. Alterations in the "pain matrix" in the brain together with alterations in the descending control of nociception [5-9] suggest that CWP/FMS is a central hyperexcitability pain condition $[8,10,11]$. Some studies, however, have found that central alterations may be driven by peripheral alterations in muscles $[3,12]$ and/or in small fibres (A-delta and C-fibre) $[13,14]$.

Today, chronic pain is assessed using subjective and semi-objective methods. The lack of validated objective markers for involved pathophysiological nociceptive processes in chronic pain conditions makes diagnosis 
problematic, so treatments are often chosen based on a trial-and-error. However, emerging evidence suggests that a bidirectional crosstalk between nociceptor neurons and the immune system play a substantial role in the modulation of chronic pain [15-19], which makes molecular components of the immune system potential markers of persistent pain states.

Bioactive endogenous lipids - e.g., endocannabinoids (ECs) and $\mathrm{N}$-acylethanolamines (NAEs) - are modulators of the immune system $[20,21]$ and influence pain and inflammation in the peripheral nervous system [22]. The NAE palmitoylethanolamide (PEA) activates peroxisome proliferator-activated receptor type- $\alpha$ (PPAR- $\alpha)$ [23], and exerts anti-inflammatory, analgesic, and neuroprotective actions [24]. Oleoylethanolamide (OEA) has anorexic properties [25] and regulates feeding and body weight through activation of PPAR- $\alpha$ [26]. OEA is also associated with both analgesic properties that may occur independently of PPAR- $\alpha$ activation [27] and with the induction of visceral pain via transient receptor potential vanilloid-1 activation [28]. Both PEA and OEA may modulate the excitability of peripheral nociceptors [22, 29]. Stearoylethanolamide (SEA) has been proposed to activate PPAR$\gamma$ [30] and to generate anti-inflammatory activity [31].

PPARs are nuclear receptors that are modulators of immune and inflammatory reactions [32]. They are involved in regulation of transcription factors and the expression of genes through a process called transrepression [33]. Both PPAR- $\alpha$ and $-\gamma$ activation inhibit the transcriptional activity of nuclear factor kappa beta (NF$\kappa B$ ), the activator protein-1 (AP-1) [34, 35], and inflammatory gene expression $[35,36]$. Activation of PPAR- $\alpha$ globally suppresses the interleukin-6 (IL-6)-stimulated acute phase response gene expression in mice [37]. PPAR- $\gamma$ agonists blocks the production of tumor necrosis factor- $\alpha$ (TNF- $\alpha$ ), IL-6, and interleukin-1 $\beta$ (IL-1 $\beta$ ) [36]. A rheumatoid arthritis study found that IL-6 and interleukin-8 (IL-8) were down regulated in synovial fibroblasts and primary synoviocytes after treatment with OEA, PEA, and arachidonoylethanolamide [38]. In rats, systemic administration of OEA and PEA reduces plasma and brain levels of TNF- $\alpha-m R N A$ induced by lipopolysaccharides [39], and SEA suppresses serum levels of TNF- $\alpha$ by inhibiting NF- $\mathrm{kB}$ translocation, which may occur via PPAR- $\gamma[30]$.

Cytokines are small non-structural proteins involved in modulation of the immune system and are generally classified as pro- or anti-inflammatory. Plasma and serum levels of cytokines have been investigated in respect to their association with CWP/FMS, but the results are inconclusive. High plasma levels of IL-8, IL-10, and TNF$\alpha$ in FMS compared to controls have been reported [40]. When investigating serum levels of IL-6, IL-8, IL-10, and TNF- $\alpha$, one study found significant higher levels of IL-10 in FMS but no alterations in the other investigated cytokines [41]. A meta-analysis concluded that plasma levels of IL-6 and IL-8 from patients with FMS were elevated [42].

We have previously reported significantly higher levels of NAEs in microdialysate samples collected from trapezius muscles in patients with CWP [43]. This study investigates systemic (plasma) levels of NAEs and cytokines in the same cohort. The primary aim was to investigate whether levels of anti-inflammatory lipid mediators OEA, PEA, and SEA, the pro-inflammatory cytokines TNF- $\alpha$, IL- $1 \beta$, IL- 6 , and IL-8, and the anti-inflammatory cytokine IL-10 differed significantly between healthy subjects and patients with CWP. Within this aim, correlations between levels of these substances and pain intensity were investigated.

\section{Methods}

\section{Subjects}

Results concerning NAEs in muscle tissue from the cohort investigated in this study (not exactly the same number) has previously been published [43, 44]. A total of 43 participants (19 CWP and 24 healthy controls $(\mathrm{CON}))$ were recruited in the original study. Blood plasma of adequate quality (haemolytic plasma were excluded) were obtainable from $17 \mathrm{CWP}$ and $21 \mathrm{CON}$ and were included in this study. The CWP subjects were identified and recruited via a fibromyalgia patient organization and by reviewing the medical reports of former out-patients at the multidisciplinary Pain and Rehabilitation Centre, University Hospital, Linköping, Sweden. Inclusion criteria were female sex, age range 20-65 years, and widespread pain according to the American College of Rheumatology 1990 classification criteria [45]. Exclusion criteria were bursitis, disorders of the spine, tendonitis, capsulitis, postoperative conditions in neck/shoulder area, prior neck trauma, neurological disease, rheumatoid arthritis or any other systemic disease, metabolic disease, malignancy, severe psychiatric illness, pregnancy, and difficulties understanding the Swedish language. All participants were examined by a standardized and validated clinical examination of the upper extremeties according to Ohlsson et al. [46].

The CON subjects were recruited via advertisements in a local daily newspaper. Inclusion criteria were female sex, age range $20-65$ years, and pain-free. The exclusion criteria were the same as the CWP group with the additional exclusion citerium of pain lasting more than 7 days during the past 12 months. Clinical examination was conducted as mentioned above.

\section{Pain intensity}

The subjects were asked to estimate their whole body pain intensity on a 10-point numeric rating scale (NRS) with the following two end points: $0=$ no pain and $10=$ worst possible pain. The results concerning pain 
intensity have essentially (although not the same number of subjects) been published elsewhere [47].

\section{Blood sampling}

Venous blood samples were drawn and centrifuged for $15 \mathrm{~min}\left(1500 \mathrm{~g}, 4 \mathrm{C}^{\circ}\right)$. Subsequently, the plasma was aliquoted $(250 \mu \mathrm{L})$ in micro centrifuge tubes and stored at $-70{ }^{\circ} \mathrm{C}$.

\section{Measurements of NAEs}

A liquid chromatography tandem mass spectrometry (LC-MS/MS) method was used to analyse NAEs in human plasma samples based on a previously published method [48]. Before measurements were taken, lipids were extracted from plasma following a previously described protocol [49]. Plasma samples were thawed and vortexed, and $25 \mu \mathrm{L}$ of deuterated internal standard AEA-d4 $(50 \mathrm{nM})$ ) were added to each plasma and blank sample. Acetonitrile $(\mathrm{ACN})(1200 \mu \mathrm{L})$ was added before vortexing and centrifugation $\left(10,000 \mathrm{rpm}, 5 \mathrm{~min}, 4{ }^{\circ} \mathrm{C}\right)$. Supernatants were added to $4.5 \mathrm{~mL}$ of milliQ- $\mathrm{H}_{2} \mathrm{O}$ containing $0.133 \%$ TFA (triflouro acetic acid). C8 Octyl SPE columns (6 mL, $200 \mathrm{mg}$ ) (Biotage; Uppsala, Sweden) were activated with 1-ml methanol and washed with $1-\mathrm{ml}$ millQ- $\mathrm{H}_{2} \mathrm{O}$ before the samples were added. After washing with $1.5-\mathrm{ml} \mathrm{ACN}$ (20\% with $0.1 \%$ TFA), the samples were eluted with 1.5 $\mathrm{ml}$ ACN ( $80 \%$ with $0.1 \%$ TFA), and dried by SpeedVacc. On the day of analysis, samples were reconstituted in $25 \mu \mathrm{L}$ of LC mobile phase. The injection volume was $10 \mu \mathrm{L}$. All standards and internal standard were purchased from Cayman Chemicals (Ann Arbor, MI, USA).

We used an HPLC-MS/MS system containing a Thermo Scientific Accela AS auto sampler and Accela 1250 pump coupled to a Thermo Scientific TSQ Quantum Access max triple quadrupole mass spectrometer with an HESI II probe as ionization source. Liquid chromatography was performed using isocratic elution on a Xbridge C8 guard column $(2.1 \mathrm{~mm} \times 10 \mathrm{~mm})$ coupled to a Xbridge C8 analytical column $(2.1 \mathrm{~mm} \times 150 \mathrm{~mm})$ both with the particle size $2.5 \mu \mathrm{m}$ obtained from Waters (Dublin, Ireland). The capillary temperature was set to $350{ }^{\circ} \mathrm{C}$ and sheath gas pressure to 40 arb units. The ion sweep gas pressure was set to 0.4 arb units. The selected reaction monitoring $(\mathrm{SRM})(\mathrm{m} / \mathrm{z})$ transitions 326.3/62.4, $300.3 / 62.4,328.3 / 62.4$ and $352.3 / 66.4$ were used for OEA, PEA, SEA, and AEA-d4 respectively. The linearity of the measuring ranges was assessed with standard curves ranging from 5 to $1000 \mathrm{nM}$ for all analytes in human plasma in triplicates and $\left.\mathrm{R}^{2} \quad 0.99( \pm 0.01)\right)$ for all analytes. Isotopic dilution was used for quantification of the analytes, performed according to their area ratio of the deuterated internal standard signal area. Linear regression and equal weighting were applied. Xcalibur ${ }^{\circ}$ (version
2.1, Thermo Scientific) software was used for peak integration and quantification.

\section{Measurements of cytokines}

Plasma cytokines were analysed using two different immunoassays. TNF- $\alpha$ and IL-1 $\beta$ were analysed with the commercial High Sensitivity Human Cytokine Magnetic Bead Panel Immunoassay (MILLIPLEX ${ }^{\circ}$ MAP for Luminex $^{\circ}$ xMAP1 Technology, EMD Millipore, Missouri, USA). The minimum detectable concentration (MinDC) was $0.7 \mathrm{pg} / \mathrm{mL}$ for TNF- $\alpha$ and $0.8 \mathrm{pg} / \mathrm{mL}$ for IL- $1 \beta$. Standards, blanks, and samples were analysed in duplicates and mean values were used. IL-6, IL- 8, and IL-10 were analysed using multiplex proximity extension assay, (Proseek $^{\bullet}$ Multiplex Inflammation I, Olink Bioscience, Uppsala, Sweden) per the manufacturer's instructions $[50,51]$. The unit is signal-to-background $(\mathrm{dCq})$. The results from these three cytokines have been published elsewhere [52].

\section{Statistics}

Traditional data analyses were perfomed using the IBM SPSS version 22.0 (IBM Corporation, Route 100 Somers, New York, USA) and the GraphPad Prism computer programmer version 6.03 (GraphPad Software Inc., San Diego, CA, USA). The number of patients needed to achieve sufficient power was based on the concentration of interstitial lactate of the trapezius in healthy controls and in patients with chronic trapezius myalgia reported in one of our previous studies [53]. Hence, using Power and Sample Size Calculation, ver. 3.0.229 based on the following parameters: $\mathrm{a}=0.05$, power $=0.8$, difference between groups $=1.7$, and $\mathrm{SD}=1.7$, it was found that 17 patients in each group were needed. Hence, this power analysis can be considered as post hoc calculation assuring that the number of subjects in the present study has the power to reveal relevant differences. T-test of independent samples was used for group comparisons. Bivariate correlation analyses (Pearson) was used, and a $p \leq 0.05$ was used as level of significance in all analysis.

Traditional univariate and bivariate statistical methods can quantify level changes of individual substances but disregard interrelationships between them and thereby ignore system-wide aspects. Therefore, multivariate data analysis (MVDA) using the software SIMCA 14.0 (Umetrics, Umeå, Sweden) was applied as a complement to the traditional statistical methods. Principal component analysis was used for data overview. The SIMCA tools Hotelling's $\mathrm{T}^{2}$ and distance to model in X-space were used to identify strong and moderate multivariate outliers, respectively. Orthogonal partial least squares-discriminant analysis was used for the multivariate regression analysis of 
group membership (CWP or CON) [54] using the levels of the investigated lipids, cytokines, and pain ratings.

For all MVDA analyses, data were log-transformed when needed using the auto transform function, and scaling to unit variance was applied $[55,56]$. The parameters $\mathrm{R}^{2}, \mathrm{Q}^{2}$, and CV-ANOVA diagnostic were used to evaluate model quality. $\mathrm{R}^{2}$ describes the goodness of fit - the fraction of sum of squares of all the variables explained by a principal component [54] $\left(\mathrm{R}^{2}=1\right.$ explains $100 \%$ of the data). $Q^{2}$ describes the goodness of prediction - the fraction of the total variation of the variables that can be predicted by a principal component using CV methods. $\mathrm{R}^{2}$ should not be considerably higher than $\mathrm{Q}^{2}$. A difference greater than $0.2-0.3$ implies overfitting, indicating poor robustness of the model is poor [55]. The CVANOVA diagnostic corresponds to a hypothesis test of the null hypothesis of equal cross-validated predictive residuals of the two compared models [57], measures the significance of the observed group separation, and returns a statistically significant $p$-value [55]. The variable influence on projection (VIP) indicates the relevance of each X-variable pooled over all dimensions and the Yvariables - the group of variables that best explains Y. VIP $\geq 1.0$ was considered significant. Coefficients (OPLS scaled and centred regression coefficients) were used to note the direction of the relationship, positive or negative (in the text this is reported immediately after the VIP value).

\section{Results}

\section{Background data}

No significant group differences in age (years) (CWP: $41.7 \pm 10$ vs. CON: $47.9 \pm 9.6)$ or body mass index (BMI) $\left(\mathrm{kg} / \mathrm{m}^{2}\right)(\mathrm{CWP}: 24.2 \pm 2.1$ vs. CON: $26.8 \pm 5.3)$ were found.

\section{Pain intensity}

The pain intensity was significantly higher in CWP $(5.3 \pm 2.1)$ compared to controls $(0.0 \pm 0.0)(p$-value $<0.001)$.

\section{NAE concentrations}

Levels of NAEs were measured in all samples (Table 1). The levels and variation of NAEs are illustrated in Fig. 1. OEA and PEA levels were significantly higher in CWP

Table 1 Mean values and standard deviations for concentrations $(n M)$ of oleoylethanolamide (OEA), palmitoylethanolamide (PEA), stearoylethanolamide (SEA) in subjects with chronic wide spread pain (CWP) and healthy controls (CON)

\begin{tabular}{llll}
\hline NAEs & CWP $(n=17)$ & CON $(n=21)$ & $p$-value \\
\hline OEA & $11.1(3.0)$ & $7.5(3.7)$ & $0.003^{*}$ \\
PEA & $18.1(9.7)$ & $10.5(6.2)$ & $0.006^{*}$ \\
SEA & $38.6(28.7)$ & $27.2(20.7)$ & 0.164 \\
\hline
\end{tabular}

"indicates statistical significance than in CON. A tendency for higher SEA levels was found in CWP, but this trend did not reach statistical significance.

\section{Cytokine concentrations}

The Luminex ${ }^{\oplus}$ assay measured TNF- $\alpha$ levels in all samples and IL- $1 \beta$ was measured in $21 \%$ of the samples (17 CWP; $20 \mathrm{CON})$. Levels under MinDC were substituted to MinDC/2. The Proseek ${ }^{\ominus}$ assay measured IL-6, IL-8, and IL-10 (presented elsewhere [52]) levels in all samples (16 CWP; $18 \mathrm{CON}$ ). No significant group differences in levels were found for the following cytokines: TNF- $\alpha(\mathrm{pg} / \mathrm{mL})$ (CWP: $5.6 \pm 1.7$ vs. CON: $5.8 \pm 3.6)$; IL-1 $(\mathrm{pg} / \mathrm{mL})$ (CWP: $0.6 \pm 0.5$ vs. CON: $2.1 \pm 6.0)$, IL-6 (dCq) (CWP: $2.7 \pm 1.7$ vs. CON: $1.9 \pm 1.5$ ); IL-8 (dCq)) (CWP: $29.8 \pm 25.8$ vs. CON: $19.0 \pm 5.9$ ); and IL-10 (dCq) (CWP: $3.2 \pm 1.0$ vs. $\mathrm{CON}: 3.0 \pm 0.6)$.

\section{Correlation analysis}

No correlation between NAEs and cytokines existed in the cohort as a whole or in the two groups analysed separately. When all participants $(\mathrm{CWP}+\mathrm{CON})$ were included, significant correlations existed between OEA and pain intensity $(r=0.49, p<0.01)$. When this was the case, the two groups were tested separately. In CWP, a significant negative correlation existed between TNF- $\alpha$ and pain intensity $(r=-0.50, p<0.05)$. Interestingly, significant inter-correlations existed between the lipids (OEA vs. PEA, $r=0.78, p<0.01$; OEA vs. SEA, $r=0.61 p<0.01$; PEA vs. SEA, $(r=0.92 p<0.01)$ in CON, although in CWP a significant correlation only existed between PEA vs. SEA $(r=0.93, p<0.01)-$ i.e., no correlation existed between OEA and PEA $(r=-0.05)$ or OEA and SEA, $(r=-0.27)$. In Fig. 2, inter-correlations of the lipids are illustrated with scatterplots of the lipid levels in each group (CON, CWP). The linearity of each plot is illustrated by the best fitted regression line $\left(R^{2}\right)$ to the scatter.

\section{The relative importance of the lipids and cytokines - multivariate analyses}

The data were primarily overviewed by a principal component analysis model to check for multivariate outliers - 38 observations (CWP and CON) and nine variables (NAEs, cytokines and pain intensity). No strong or moderate outlier was found by Hoteling's $\mathrm{T}^{2}$ statistics ( $\mathrm{T}^{2}$ Critical 99\%) and distance to model in Xspace (DCrit (0.05)).

In the following step, levels of NAEs, cytokines, and pain intensity were used for OPLS-DA regression of group membership. A strong significant regression model was obtained $\left(\mathrm{R}^{2}=0.93, \mathrm{Q}^{2}=0.89\right.$, and CV-ANOVA: $P<0.001)$. As expected, pain intensity was the most important regressor, VIP $=1.80(+)$. Interestingly, OEA 


\section{OEA}

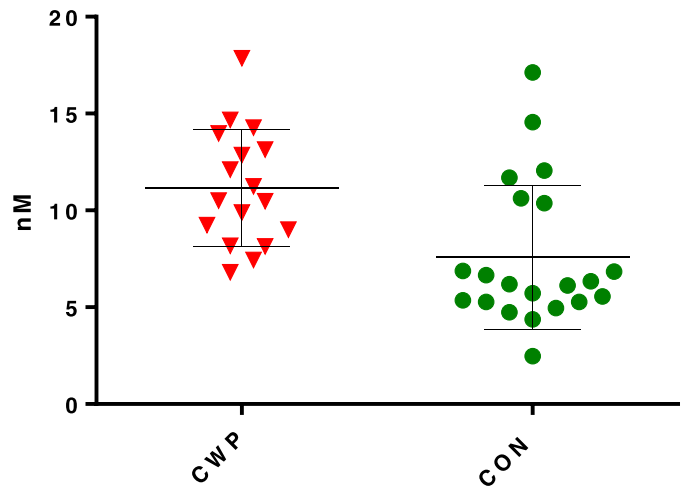

$\nabla$ CW P

- $\operatorname{CON}$

PEA

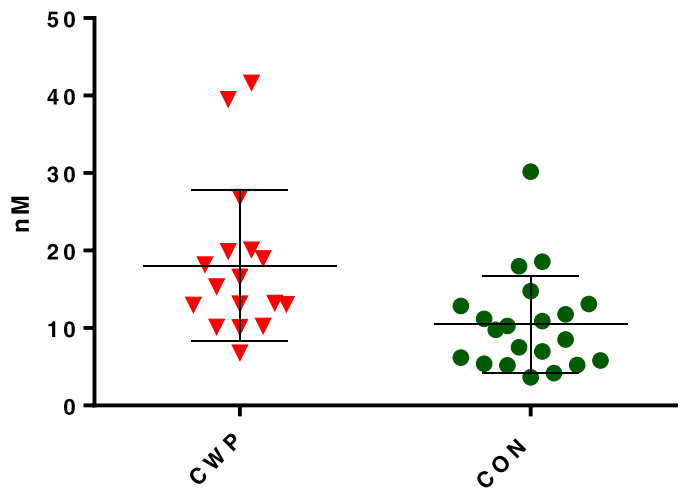

$\nabla$ CW P

- $\operatorname{CON}$

SEA

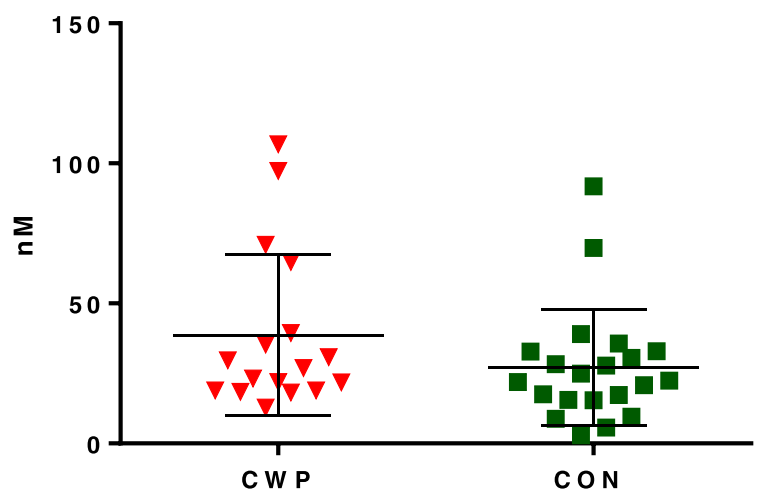

Fig. 1 Scatter plots of concentrations (nM) in plasma for oleoylethanolamide (OEA), palmitoylethanolamide (PEA), and stearoylethanolamide (SEA) sampled from women with chronic wide spread pain (CWP) and healthy female controls (CON). The horizontal lines illustrate the group mean (middle-line) with error bars ( \pm SD) above and below

and PEA were also important regressors, VIP $=1.19(+)$ and $1.17(+)$, respectively. Cytokines, SEA, BMI, and age had VIP $<0.9$ and were relatively unimportant regressors in that model.

In the next step, only NAEs and Cytokines were used as regressors. A weaker yet significant model was obtained $\left(\mathrm{R}^{2}=0.32, \mathrm{Q}^{2}=0.26\right.$, and CV-ANOVA: $\left.P=0.012\right)$. Most important regressors in that model were PEA (VIP $=1.57$ $(+))$, OEA (VIP $=1.47(+))$, and SEA (VIP $=1.30(+)$ ). Hence, when inter-correlations between NAEs and cytokines were also taken into consideration (i.e., in contrast to the traditional statistical analyses), all three NAEs significantly contributed to the between group differentiation and thus were higher in CWP than CON. All cytokines 


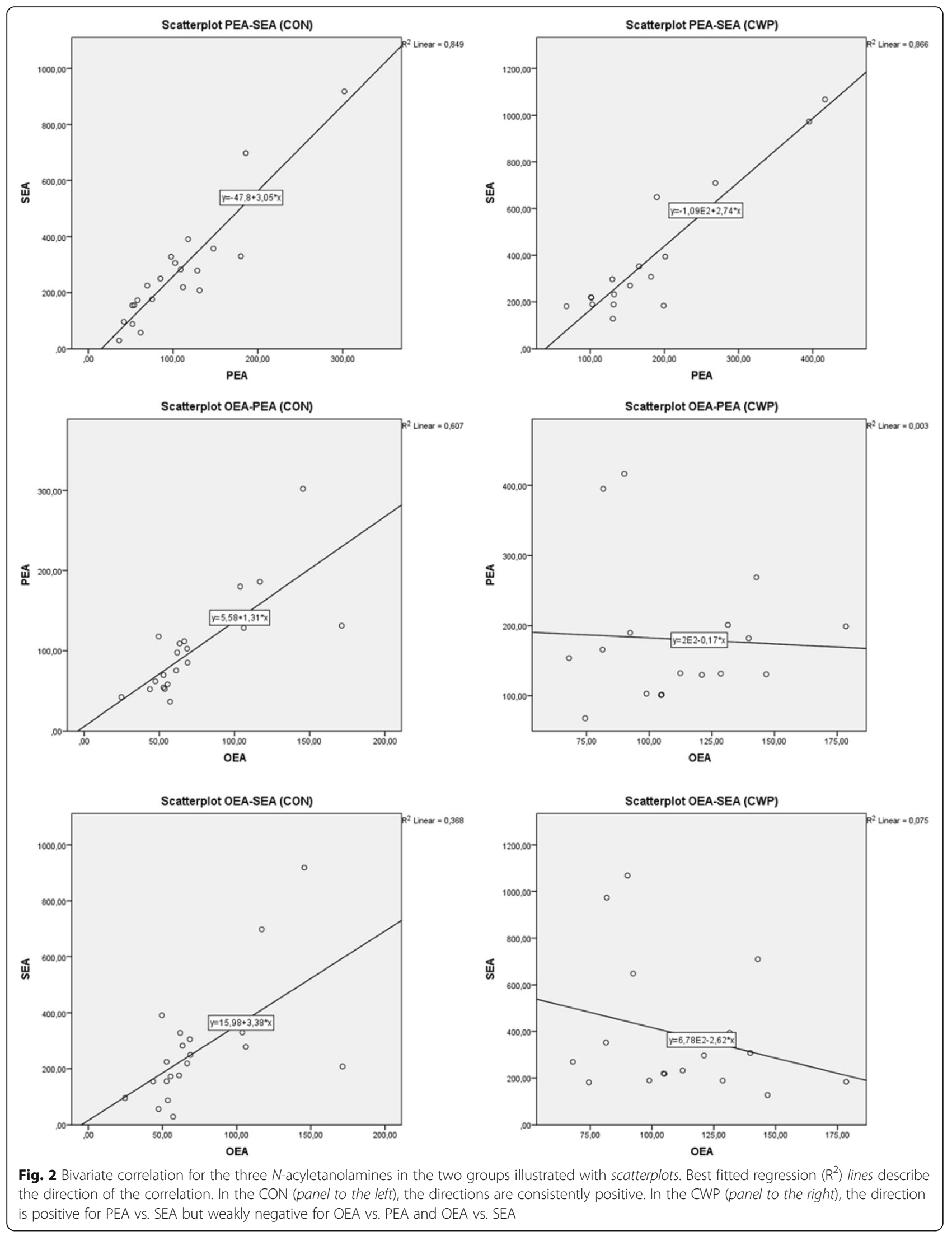


had VIP $<0.86$, so they were relatively unimportant regressors compared to the NAEs with respect to group belonging. In Fig. 3, VIP values of all substances are represented as bars with error bars.

\section{Discussion}

This study revealed three major findings.

- Significantly higher plasma levels of NAEs and especially for OEA and PEA were found in CWP.

- Multivariate regression modelling confirmed that cytokines were relatively unimportant compared to NAEs with respect to group belonging.

- No significant correlations existed between cytokines and NAEs.

A previous investigation of this cohort found that the CWP group had higher NAE levels in microdialysate locally sampled from the trapezius muscle during the first $2 \mathrm{~h}$ after microdialysis probe insertion [43]. These results indicate that levels of NAEs are altered in CWP although with some differences between microdialysate and plasma levels. In the microdialysate samples, SEA and OEA (not PEA) were significantly higher, but alterations of OEA and PEA (not SEA) were significantly higher in plasma. Our results taken together may reflect an altered NAE metabolism in CWP compared to CON.
To the best of our knowledge, there are only two previous studies that have investigated plasma levels of NAEs in CWP/FMS. High levels of the endocannabinoid arachidonoylethanolamide were reported in patients with FMS compared to controls [58]. Recently, Hellström et al. investigated the levels of OEA, PEA, SEA, and other related lipids in 15 woman with CWP and 27 healthy controls [59], but they did not find statistically significant group differences. A possible explanation for the different findings between their study and our study could be the selection of patients. Although both studies used the American College of Rheumatology CWP criteria from 1990, most patients (15/17) in ours were classified as FMS patients and had a NRS mean score of $5.3 \pm 2.1$ compared to a median NRS score of 3 for the CWP patients in Hellström et al.

In the present study, no alterations of traditionally investigated cytokines were found between groups, a finding that agrees with some reports and disagrees with other reports [40-42]. A significant negative correlation between TNF- $\alpha$ and pain intensity in CWP existed, which (to the best of our knowledge) has not been previously reported. Wang et al. found elevated levels of circulating TNF- $\alpha$ in FMS, but they did not observe any correlation between these levels and pain intensity [60]. In a more recent study, Christidis et al. did not find any difference between plasma levels of TNF- $\alpha$ between FMS

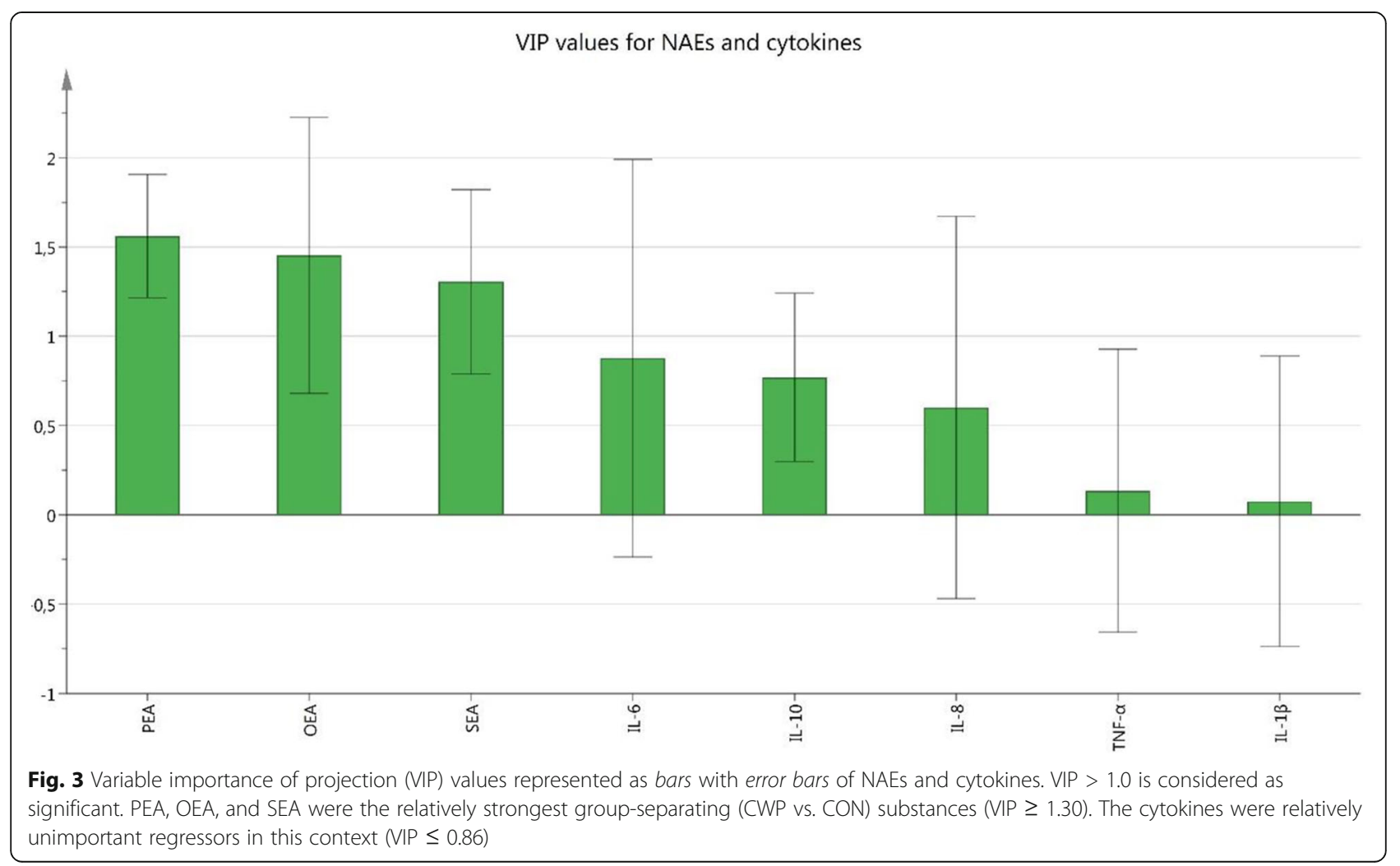


and controls and did not find any correlation with pain intensity [61].

NAEs under investigation in this study have been proposed to suppress levels of various cytokines [30, 38, 39]. However, we found no correlations between cytokine and NAE levels. Another observation was that all NAEs positively inter-correlated significantly in CON. In CWP, a significant positive correlation existed only between PEA and SEA (Fig. 2). This difference in patterns between the two groups together with the group alterations indicates an altered NAE metabolism in CWP; however, the possible cause and relevance of such alteration needs more investigation. Our results concerning NAEs together with results from a panel of inflammatory proteins from the same cohort [52] and results from another cohort [62] may indicate that systemic inflammation is present in CWP.

By using MVDA, we confirmed the traditional statistical analyses. When all participants (CWP and CON) were included, significant models were obtained both with and without the pain intensity variable included. In those models, OEA and PEA were the most important (group separating) substances.

Because we already reported on elevated levels of NAEs in CWP from locally sampled trapezius muscles [43], the main strength of this study is that it measures systemic levels in the same cohort. The results confirm that systemic NAE levels are altered as well, which increases the data's impact. Another strength of our study is that it compares the impact of cytokines potentially related to CWP with NAEs with respect to group belonging (CWP or CON). A majority of NAEs, including OEA, PEA, and SEA, have been reported to be influenced by diet $[63,64]$ and both PEA and OEA are associated with energy metabolism [65]. Therefore, one limitation of this study is that dietary factors were not captured. In addition, our study investigated a limited number of molecular components and our sample size was relatively small.

\section{Conclusions}

The nociceptive and the immune systems are linked. The systems are specialized to prevent tissue damage and restore homeostasis by targeting and modulating harmful stimuli.

In this study, two classes of molecules associated with the nociceptive and immune systems were investigated (NAEs and cytokines) in CWP and in CON. Altered levels and altered relative composition of NAEs were found in CWP compared to CON. As partly reported elsewhere, no alterations of cytokine levels were found and no correlations existed between NAEs and cytokines. Circulating levels of NAEs may indicate systemic inflammation in CWP better than the investigated cytokines; however, more studies are needed.

\section{Abbreviations}

CON: Healthy controls; CWP: Chronic widespread pain; FMS: Fibromyalgia syndrome; IL-10: Interleukin-10; IL-1ß: Interleukin-1 beta; IL-6: Interleukin-6; IL-8: Interleukin-8; NAE: N-acylethanolamines; NRS: Numeric rating scale; OEA: Oleoylethanolamide; OPLS-DA: Orthogonal partial least squaresdiscriminant analysis; PEA: Palmitoylethanolamide; SEA: Stearoylethanolamide; TNF-a: Tumour necrosis factor-alpha; VIP: Variable influence on projection

\section{Acknowledgements}

Not applicable.

\section{Funding}

This study was funded by the Swedish Research Council (K2015-99x-21,874-05-4), Swedish Council for Working Life and Social Research (2010-0913), AFA Insurance, the Region Östergötland foundation, and Åke Wiberg foundation.

\section{Availability of data and materials}

The datasets used and analysed during the current study are available from the corresponding author on reasonable request.

\section{Authors' contributions}

Design of the study: all authors; Clinical examination and blood sampling: NG; biochemical analyses: NS and BGh; statistical analyses: NS and BGe; first draft of the manuscript: NS; comments on drafts of the manuscript: all authors; approval of the final draft: all authors.

\section{Competing interests}

The authors declare that they have no competing interests.

\section{Consent for publication}

Not applicable.

\section{Ethics approval and consent to participate}

All participants in this study signed a consent form that was in accordance with the Declaration of Helsinki. The study was granted ethical clearance by the Linköping University Ethics Committee (Dnr: M10-08., M233-09, M23309; Dnr: 2010/164-32).

\section{Publisher's Note}

Springer Nature remains neutral with regard to jurisdictional claims in published maps and institutional affiliations.

Received: 4 April 2017 Accepted: 30 May 2017

Published online: 12 June 2017

\section{References}

1. Mansfield KE, et al. A systematic review and meta-analysis of the prevalence of chronic widespread pain in the general population. Pain. 2016;157(1):55-64.

2. Gran JT. The epidemiology of chronic generalized musculoskeletal pain. Best Pract Res Clin Rheumatol. 2003;17(4):547-61.

3. Staud R. Peripheral pain mechanisms in chronic widespread pain. Best Pract Res Clin Rheumatol. 2011;25(2):155-64.

4. Gerdle B, et al. Altered neuromuscular control mechanisms of the trapezius muscle in fibromyalgia. BMC Musculoskelet Disord. 2010;11:42.

5. Price DD, et al. Enhanced temporal summation of second pain and its central modulation in fibromyalgia patients. Pain. 2002;99(1-2):49-59.

6. Desmeules JA, et al. Neurophysiologic evidence for a central sensitization in patients with fibromyalgia. Arthritis Rheum. 2003;48(5):1420-9.

7. DeSantana JM, Sluka KA. Central mechanisms in the maintenance of chronic widespread noninflammatory muscle pain. Curr Pain Headache Rep. 2008; 12(5):338-43.

8. Petersel DL, Dror V, Cheung R. Central amplification and fibromyalgia: disorder of pain processing. J Neurosci Res. 2011;89(1):29-34

9. Jensen $\mathrm{KB}$, et al. Patients with fibromyalgia display less functional connectivity in the brain's pain inhibitory network. Mol Pain. 2012;8:32.

10. Bradley LA. Pathophysiology of fibromyalgia. Am J Med. 2009;122(12 Suppl): S22-30

11. Smith H, Harris R, Clauw D. Fibromyalgia: an afferent processing disorder leading to a complex pain generalized syndrome. Pain Physician. 2011; 14(2):E217-45. 
12. Staud $\mathrm{R}$, et al. Enhanced central pain processing of fibromyalgia patients is maintained by muscle afferent input: a randomized, double-blind, placebocontrolled study. Pain. 2009;145(1-2):96-104.

13. Serra J, et al. Hyperexcitable $\mathrm{C}$ nociceptors in fibromyalgia. Ann Neurol. 2014;75(2):196-208.

14. Uceyler $\mathrm{N}$, et al. Small fibre pathology in patients with fibromyalgia syndrome. Brain. 2013;136(Pt 6):1857-67

15. Verma V, Sheikh Z, Ahmed AS. Nociception and role of immune system in pain. Acta Neurol Belg. 2015;115(3):213-20.

16. Marchand F, Perretti M, McMahon SB. Role of the immune system in chronic pain. Nat Rev Neurosci. 2005;6(7):521-32.

17. Generaal $E$, et al. Basal inflammation and innate immune response in chronic multisite musculoskeletal pain. Pain. 2014;155(8):1605-12.

18. Pinho-Ribeiro FA, Verri WA Jr, Chiu IM. Nociceptor sensory neuron-immune interactions in pain and inflammation. Trends Immunol. 2017:38(1):5-19.

19. McMahon SB, La Russa F, Bennett DL. Crosstalk between the nociceptive and immune systems in host defence and disease. Nat Rev Neurosci. 2015 16(7):389-402.

20. Cabral GA, Ferreira GA, Jamerson MJ. Endocannabinoids and the immune system in health and disease. Handb Exp Pharmacol. 2015;231:185-211.

21. Skaper SD, et al. N-Palmitoylethanolamine and Neuroinflammation: a nove therapeutic strategy of resolution. Mol Neurobiol. 2015;52(2):1034-42.

22. Piomelli D, Sasso O. Peripheral gating of pain signals by endogenous lipid mediators. Nat Neurosci. 2014;17(2):164-74.

23. Lo Verme J, et al. The nuclear receptor peroxisome proliferator-activated receptor-alpha mediates the anti-inflammatory actions of palmitoylethanolamide. Mol Pharmacol. 2005;67(1):15-9.

24. Alhouayek M, Muccioli GG. Harnessing the anti-inflammatory potential of palmitoylethanolamide. Drug Discov Today. 2014;19(10):1632-9.

25. Rodriguez de Fonseca F, et al. An anorexic lipid mediator regulated by feeding. Nature. 2001;414(6860):209-12

26. Fu J, et al. Oleylethanolamide regulates feeding and body weight through activation of the nuclear receptor PPAR-alpha. Nature. 2003;425(6953):90-3.

27. Suardiaz $M$, et al. Analgesic properties of oleoylethanolamide (OEA) in visceral and inflammatory pain. Pain. 2007;133(1-3):99-110

28. Wang X, Miyares RL, Ahern GP. Oleoylethanolamide excites vagal sensory neurones, induces visceral pain and reduces short-term food intake in mice via capsaicin receptor TRPV1. J Physiol. 2005;564(Pt 2):541-7.

29. Piomelli $D$, et al. A lipid gate for the peripheral control of pain. J Neurosci. 2014:34(46):15184-91.

30. Berdyshev AG, et al. N-Stearoylethanolamine suppresses the pro-inflammatory cytokines production by inhibition of NF-kappaB translocation. Prostaglandins Other Lipid Mediat. 2015;121:91

31. Dalle Carbonare $\mathrm{M}$, et al. A saturated $\mathrm{N}$-acylethanolamine other than $\mathrm{N}$ palmitoyl ethanolamine with anti-inflammatory properties: a neglected story. J Neuroendocrinol. 2008;20(Suppl 1):26-34.

32. Zhang $X$, Young HA. PPAR and immune system-what do we know? Int Immunopharmacol. 2002;2(8):1029-44.

33. Ricote M, Glass CK. PPARs and molecular mechanisms of transrepression. Biochim Biophys Acta. 2007;1771(8):926-35.

34. Ricote $\mathrm{M}$, et al. The peroxisome proliferator-activated receptor-gamma is a negative regulator of macrophage activation. Nature. 1998;391(6662):79-82.

35. Delerive $P$, et al. Peroxisome proliferator-activated receptor alpha negatively regulates the vascular inflammatory gene response by negative cross-talk with transcription factors NF-kappaB and AP-1. J Biol Chem. 1999;274(45):32048-54.

36. Jiang C, Ting AT, Seed B. PPAR-gamma agonists inhibit production of monocyte inflammatory cytokines. Nature. 1998;391(6662):82-6.

37. Gervois $P$, et al. Global suppression of IL-6-induced acute phase response gene expression after chronic in vivo treatment with the peroxisome proliferatoractivated receptor-alpha activator fenofibrate. J Biol Chem. 2004;279(16):16154-60.

38. Lowin $\mathrm{T}$, et al. Anti-inflammatory effects of $\mathrm{N}$-acylethanolamines in rheumatoid arthritis synovial cells are mediated by TRPV1 and TRPA1 in a COX-2 dependent manner. Arthritis Res Ther. 2015;17:321.

39. Sayd A, et al. Systemic administration of oleoylethanolamide protects from neuroinflammation and anhedonia induced by LPS in rats. Int J Neuropsychopharmacol. 2015;18(6):1-14.

40. Bazzichi $L$, et al. Cytokine patterns in fibromyalgia and their correlation with clinical manifestations. Clin Exp Rheumatol. 2007;25(2):225-30.

41. Ranzolin A, et al. Evaluation of cytokines, oxidative stress markers and brainderived neurotrophic factor in patients with fibromyalgia - a controlled cross-sectional study. Cytokine. 2016;84:25-8.
42. Uceyler N, Hauser W, Sommer C. Systematic review with meta-analysis: cytokines in fibromyalgia syndrome. BMC Musculoskelet Disord. 2011;12:245.

43. Stensson $N$, et al. High levels of endogenous lipid mediators ( $N$ acylethanolamines) in women with chronic widespread pain during acute tissue trauma. Mol Pain. 2016;12:1-11.

44. Ghafouri N, et al. Palmitoylethanolamide and stearoylethanolamide levels in the interstitium of the trapezius muscle of women with chronic widespread pain and chronic neck-shoulder pain correlate with pain intensity and sensitivity. Pain. 2013;154(9):1649-58.

45. Wolfe F, et al. The American College of Rheumatology 1990 criteria for the classification of fibromyalgia. Report of the multicenter criteria committee. Arthritis Rheum. 1990;33(2):160-72.

46. Ohlsson $\mathrm{K}$, et al. An assessment of neck and upper extremity disorders by questionnaire and clinical examination. Ergonomics. 1994;37(5):891-7.

47. Gerdle B, et al. Chronic widespread pain: increased glutamate and lactate concentrations in the trapezius muscle and plasma. Clin J Pain. 2014;30(5):409-20.

48. Stensson $\mathrm{N}$, et al. Identification of lipid mediators in peripheral human tissues using an integrative in vivo microdialysis approach. J Anal Bioanal Tech. 2016;7:306.

49. Balvers MG, Verhoeckx KC, Witkamp RF. Development and validation of a quantitative method for the determination of 12 endocannabinoids and related compounds in human plasma using liquid chromatography-tandem mass spectrometry. J Chromatogr B Analyt Technol Biomed Life Sci. 2009; 877(14-15):1583-90.

50. Assarsson $\mathrm{E}$, et al. Homogenous 96-plex PEA immunoassay exhibiting high sensitivity, specificity, and excellent scalability. PLoS One. 2014;9(4):e95192.

51. Moen A, et al. Inflammatory serum protein profiling of patients with lumbar radicular pain one year after disc herniation. Int J Inflam. 2016;2016:3874964

52. Gerdle B, et al. Signs of inflammation in female patients with chronic widespread pain-a multivariat, explorative, cross-sectional study of blood samples. Med. 2017. in press.

53. Rosendal $L$, et al. Increase in muscle nociceptive substances and anaerobic metabolism in patients with trapezius myalgia: microdialysis in rest and during exercise. Pain. 2004;112(3):324-34.

54. Eriksson L, et al. Multi- and Megavariate data analysis; part I and II. 2nd ed. Umeå: Umetrics AB; 2006.

55. Wheelock AM, Wheelock CE. Trials and tribulations of 'omics data analysis: assessing quality of SIMCA-based multivariate models using examples from pulmonary medicine. Mol BioSyst. 2013;9(11):2589-96.

56. Eriksson L, et al. Multi- and Megavariate data analysis: basic principles and applications. Third revised edition ed. Malmö: MKS Umetrics AB; 2013.

57. Eriksson L, Trygg J, Wold S. CV-ANOVA for significance testing of PLS and OPLS ${ }^{\circledast}$ models. J Chemom. 2008;22(11-12):594-600.

58. Kaufmann I, et al. Anandamide and neutrophil function in patients with fibromyalgia. Psychoneuroendocrinology. 2008;33(5):676-85.

59. Hellstrom $F$, et al. Association between plasma concentrations of linoleic acid-derived oxylipins and the perceived pain scores in an exploratory study in women with chronic neck pain. BMC Musculoskelet Disord. 2016:17:103.

60. Wang $\mathrm{H}$, et al. Circulating cytokine levels compared to pain in patients with fibromyalgia - a prospective longitudinal study over 6 months. J Rheumatol. 2008;35(7):1366-70.

61. Christidis N, et al. Comparison of the levels of pro-inflammatory cytokines released in the Vastus Lateralis muscle of patients with fibromyalgia and healthy controls during contractions of the quadriceps muscle-a microdialysis study. PLoS One. 2015;10(12):e0143856.

62. Bäckryd $\mathrm{E}$, et al. Evidence of both systemic inflammation and neuroinflammation in fibromyalgia patients, as assessed by a multiplex protein panel applied to the cerebrospinal fluid and to plasma. J Pain Res. 2017. in press.

63. Artmann A, et al. Influence of dietary fatty acids on endocannabinoid and $\mathrm{N}$-acylethanolamine levels in rat brain, liver and small intestine. Biochim Biophys Acta. 2008:1781(4):200-12.

64. Kleberg K, Hassing HA, Hansen HS. Classical endocannabinoid-like compounds and their regulation by nutrients. Biofactors. 2014;40(4):363-72.

65. Hansen HS. Role of anorectic N-acylethanolamines in intestinal physiology and satiety control with respect to dietary fat. Pharmacol Res. 2014:86:18-25. 